\title{
Surf early to higher tides: surfactant therapy to optimize tidal volume, lung recruitment, and iNO response
}

\author{
Girija G. Konduri $\mathbb{D}^{1} \cdot$ Satyan Lakshminrusimha $\mathbb{D}^{2}$
}

Received: 11 June 2020 / Revised: 25 June 2020 / Accepted: 22 July 2020 / Published online: 13 August 2020

(c) Springer Nature America, Inc. 2020

\begin{abstract}
Inhaled nitric oxide is approved by FDA for the management of hypoxemic respiratory failure in term and near-term infants. However, approximately a third of patients treated with inhaled nitric oxide fail to have a sustained improvement in oxygenation. Recruitment of the lung with surfactant enables optimal delivery of nitric oxide to the alveolar space leading to effective pulmonary vasodilation.
\end{abstract}

At birth the fetal lung transitions from a liquid-filled, nonrespiring organ to the sole organ performing gas exchange during postnatal life. This adaptation can fail due to a variety of conditions, including retention of fetal lung liquid, surfactant deficiency, aspiration of meconium/blood/ amniotic fluid, pneumonia/sepsis, and congenital anomalies that interfere with the establishment of ventilation at birth. Normal transition also requires a rapid decrease in pulmonary vascular resistance (PVR) to accommodate the nearly ten-fold increase in pulmonary blood flow at birth to match ventilation with perfusion [1]. As expected, conditions that interfere with the establishment of ventilation and/ or increase in pulmonary blood flow lead to hypoxemic respiratory failure (HRF). This is due to ventilationperfusion mismatch and/or persistent pulmonary hypertension of the newborn (PPHN) with right-to-left shunting of blood across patent foramen ovale and/or patent ductus arteriosus [1].

Term and late preterm infants with HRF have an increased risk of mortality. Survivors have an increased risk of neurodevelopmental impairments and post-discharge medical needs [2-5]. Parenchymal lung disease with alveolar

Satyan Lakshminrusimha

slakshmi@ucdavis.edu

1 Department of Pediatrics, Medical College of Wisconsin and Children's Research Institute, Children's Wisconsin, Milwaukee, WI 53226, USA

2 Department of Pediatrics, UC Davis School of Medicine, Sacramento, CA 95817, USA atelectasis from one of the conditions listed above occurs in $65-80 \%$ of the infants with HRF [2, 6, 7]. PPHN complicates the course of HRF in up to $75-80 \%$ of the affected infants, as determined by Echocardiography [2]. Infants without parenchymal lung disease can also develop PPHN, referred to as primary (or "black-lung") PPHN. The introduction of inhaled nitric oxide (iNO) therapy for the management of HRF/PPHN led to striking decreases in the risk of using extracorporeal membrane oxygenation (ECMO) as a rescue therapy and/or risk of mortality $[2,8]$. Inhaled NO selectively dilates the pulmonary arteries, which makes it an ideal agent for neonates with PPHN [1, 2]. Since iNO is distributed to the ventilated segments of the lung, it dilates pulmonary arteries in the ventilated open areas of the lung, improving the ventilation/perfusion matching. This microselective effect (Fig. 1a) is particularly important for term and late preterm infants with HRF, since majority of them have some degree of parenchymal lung disease. However, iNO requires sufficient alveolar surface area to diffuse across to the pulmonary vasculature in order to effectively lower the PVR (Fig. 1b). Therefore, lung recruitment and keeping the majority of alveoli open are important prerequisites to optimize response to iNO. Suboptimal lung recruitment is a key mechanism for failure of oxygenation response to iNO. Neonates who fail to respond to iNO have an increased risk of ECMO/death [9].

Surfactant deficiency is a major component of parenchymal lung disease, occurring either as the primary deficiency in respiratory distress syndrome or a secondary loss from inactivation (meconium), injury (pneumonia, inflammation), or dilution (excess fluid in transient tachypnea of newborn-TTN). Several studies over the last 

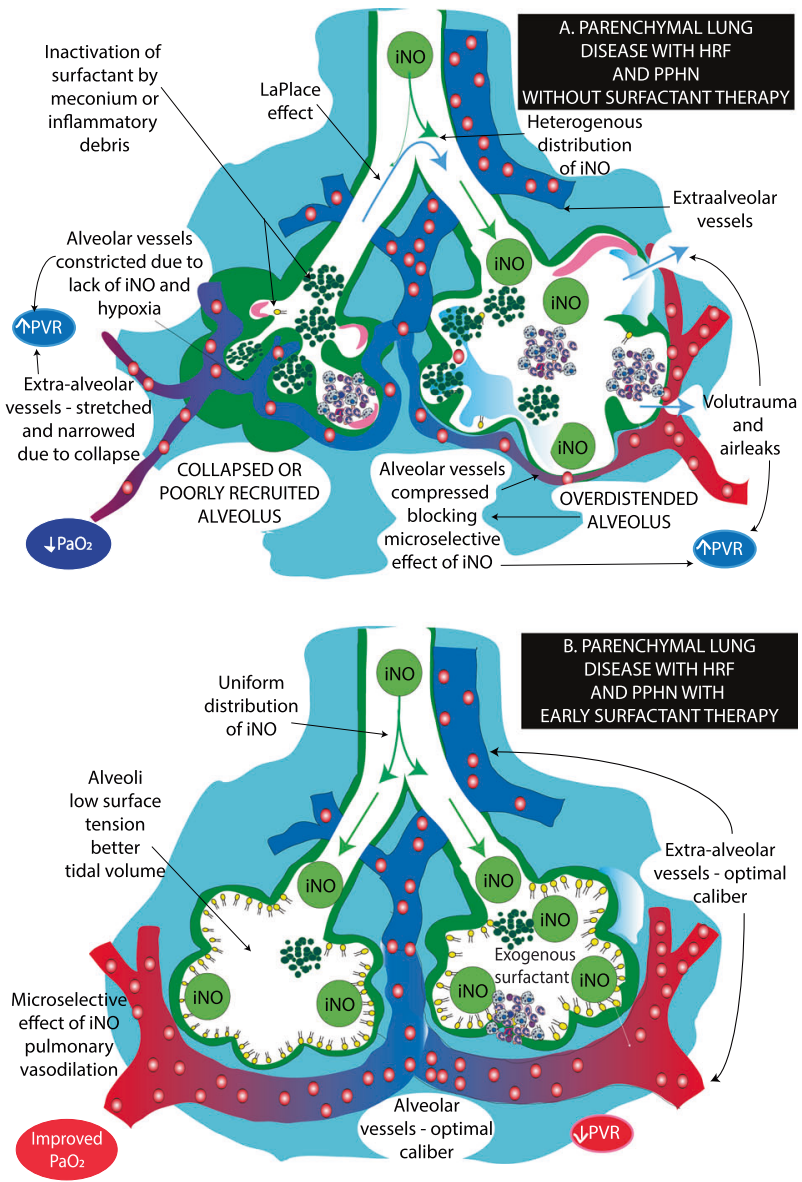

Fig. 1 Parenchymal lung disease with hypoxemic respiratory failure (HRF) and persistent pulmonary hypertension of the newborn (PPHN) in term neonates. a Asymmetric lung expansion results in collapsed and overdistended alveoli when treated with inhaled nitric oxide (iNO) without surfactant or alveolar recruitment. Due to the LaPlace effect, alveoli with smaller diameter have high pressure and empty into alveoli with larger diameter. Alveolar collapse leads to extra-alveolar pulmonary vasoconstriction. In addition, hypoxia and decreased distribution of iNO (microselective effect) lead to constriction of alveolar vessels further contributing to high pulmonary vascular resistance (PVR). Overdistension of the alveolus compresses alveolar pulmonary vessels, increasing PVR. In addition, overdistension results in volutrauma and air leaks, both of which contribute to high PVR and exacerbation of PPHN with poor response to iNO. b Treated with early surfactant and iNO: early surfactant and lung recruitment results in homogenous lung distension with effective distribution of iNO, improved tidal volumes due to increased compliance, and pulmonary vasodilation. The net result is low PVR and improved oxygenation. Copyright Satyan Lakshminrusimha and Ganesh Konduri.

25 years have demonstrated the benefit of surfactant administered to term and late preterm infants with a variety of lung diseases in mitigating the progression of respiratory failure. In a pilot randomized trial, Findlay et al. have shown that surfactant therapy improves oxygenation and decreases the need for ECMO, while reducing the number of ventilator days in neonates with meconium aspiration syndrome [10]. In a large multicenter RCT, Lotze et al. have demonstrated that a bovine surfactant given at $6 \mathrm{~h}$ intervals for up to four doses to late preterm and term infants with HRF and an oxygenation index (OI) $>15$ significantly decreased the need for ECMO, compared to a placebo [11]. A subgroup analysis of this study found that infants who presented at an OI 15-22 had the most significant reduction in the ECMO rate. Infants who presented at OI of 23-30 had a modest reduction in ECMO, while those at an $\mathrm{OI}>30$ did not show a response. In a post-hoc analysis of infants enrolled into an RCT of early iNO therapy, Konduri et al. reported that term/late preterm infants who received surfactant for moderate HRF, defined as OI of 15-25, had a significant threefold reduction in the risk of ECMO/death in the presence of parenchymal lung disease and decreased duration of oxygen use and hospital stay [8]. These data demonstrate the importance of surfactant given early in the course of HRF in improving oxygenation and decreasing the progression of HRF. However, an RCT to show the efficacy of surfactant in improving oxygenation in infants who are receiving iNO therapy has not been conducted previously.

In this Journal issue, Gonzalez et al. randomly assigned term and late preterm infants with $\mathrm{HRF}$ and an OI $>20$ to either surfactant $+\mathrm{iNO}$ or to placebo+iNO therapy [12]. The primary outcome of the study is progression of HRF to OI $>40$. This is a well-designed, prospective, randomized, double blinded, multicenter trial with carefully defined entry criteria. The presence of PPHN, identified by echocardiography was required for enrollment. The study groups were appropriately balanced with respect to background demographics and infants with different lung diseases between the two groups. Treatment guidelines were standardized. Surfactant was offered as an open label, rescue therapy for infants who progressed to OI $>40$. The investigators found that infants who received early surfactant $+\mathrm{iNO}$ improved their oxygenation earlier compared to the placebo+iNO group, and were significantly less likely to progress to OI > 40. This was the primary study outcome. Although it was not the primary outcome, surfactant treatment also significantly decreased the proportion of babies that needed ECMO and/or died. As the authors pointed out, this is the first RCT that evaluated the efficacy of surfactant in neonates who were already receiving iNO therapy. The investigators should be commended for completing this study, despite slow enrollment and nearly 5 years to reach the target sample size. Enrollment of these critically ill neonates into prospective interventional studies is challenging, as illustrated by a recent study that closed short of enrollment goals due to slow recruitment [13].

There are some limitations to the study, as pointed out by the authors. The study infants were sicker, with mean OI of 
38 , rather than the intended target OI of 20 at enrollment, and were close to the primary outcome of reaching an OI of 40. Earlier treatment may have made even a more significant impact on the progression of HRF than what was observed in this study. ECMO in this trial was provided at one tertiary care site, with three infants dying before referral could be done. In addition, $90 \%$ of the study infants had parenchymal lung disease, which contributes to a high response rate to surfactant. Previous studies have shown that infants with primary PPHN do not benefit from surfactant therapy $[8,11]$.

While clinicians have known about the physiological benefits of lung recruitment through early surfactant therapy to optimize the response to iNO, this study provides important objective evidence to support this practice. When used in conjunction with iNO, surfactant likely exerts its beneficial effects in lung disease through homogeneous lung recruitment, minimizing areas of atelectasis and overdistenstion (Fig. 1b); decrease in lung injury/inflammation; and promoting the vasodilator efficacy of iNO. The beneficial effect is very consistent across several RCTs, providing level $1 \mathrm{~A}$ evidence that early use of surfactant is the key to optimizing the response to iNO, improving oxygenation, and preventing adverse consequences of rapid progression of HRF. This approach should now be the standard practice for neonates in HRF and PPHN due to parenchymal lung disease.

\section{Compliance with ethical standards}

Conflict of interest The authors declare that they have no conflict of interest.

Publisher's note Springer Nature remains neutral with regard to jurisdictional claims in published maps and institutional affiliations.

\section{References}

1. Lakshminrusimha S. The pulmonary circulation in neonatal respiratory failure. Clin Perinatol. 2012;39:655-83.
2. Neonatal Inhaled Nitric Oxide Study Group. Inhaled nitric oxide in full-term and nearly full-term infants with hypoxic respiratory failure. N Engl J Med. 1997;336:597-604.

3. Neonatal Inhaled Nitric Oxide Study Group (NINOS). Inhaled nitric oxide in term and near-term infants: neurodevelopmental follow-up of the neonatal inhaled nitric oxide study group (NINOS). J Pediatr. 2000;136:611-7. https://doi.org/10.1067/ mpd.2000.104826

4. Konduri GG, Vohr B, Robertson C, et al. Early inhaled nitric oxide therapy for term and near-term newborn infants with hypoxic respiratory failure: neurodevelopmental follow-up. J Pediatr. 2007;150:235-40.e1. https://doi.org/10.1016/j.jpeds. 2006.11.065

5. Steurer MA, Baer RJ, Oltman S, et al. Morbidity of persistent pulmonary hypertension of the newborn in the first year of life [published correction appears in J Pediatr. 2019 Dec;215:291. Abstract corrected]. J Pediatr. 2019;213:58-65.e4. https://doi.org/ 10.1016/j.jpeds.2019.06.053

6. Truog WE, Kurth G, Haney B, Kilbride HW. Hypoxic respiratory failure: etiology and outcomes at one referral center 2000 through 2005. J Perinatol. 2007;27:371-4.

7. Steurer MA, Jelliffe-Pawlowski LL, Baer RJ, Partridge JC, Rogers EE, Keller RL. Persistent pulmonary hypertension of the newborn in late preterm and term infants in California. Pediatrics. 2017;139:e20161165. https://doi.org/10.1542/peds.2016-1165

8. Konduri GG, Sokol GM, Van Meurs KP, et al. Impact of early surfactant and inhaled nitric oxide therapies on outcomes in term/ late preterm neonates with moderate hypoxic respiratory failure. $\mathrm{J}$ Perinatol. 2013;33:944-9. https://doi.org/10.1038/jp.2013.83

9. Gupta A, Rastogi S, Sahni R, et al. Inhaled nitric oxide and gentle ventilation in the treatment of pulmonary hypertension of the newborn-a single-center, 5-year experience. J Perinatol. 2002;22:435-41. https://doi.org/10.1038/sj.jp.7210761

10. Findlay RD, Taeusch HW, Walther FJ. Surfactant replacement therapy for meconium aspiration syndrome. Pediatrics. 1996;97: $48-52$.

11. Lotze A, Mitchell BR, Bulas DI, Zola EM, Shalwitz RA, Gunkel $\mathrm{JH}$. Multicenter study of surfactant (beractant) use in the treatment of term infants with severe respiratory failure. Survanta in Term Infants Study Group. J Pediatr. 1998;132:40-7.

12. Gonzalez A, Bancalari A, Osorio W, Luco M, González A, Pérez $\mathrm{H}$, et al. Early use of combined exogenous surfactant and inhaled nitric oxide reduces treatment failure in persistent pulmonary hypertension of the newborn: a randomized controlled trial. J Perinatol. https://doi.org/10.1038/s41372-020-00777-x.

13. Steinhorn RH, Fineman J, Kusic-Pajic A, et al. Bosentan as adjunctive therapy for persistent pulmonary hypertension of the newborn: results of the randomized multicenter placebo-controlled exploratory trial. J Pediatr. 2016;177:90-96.e3. https://doi.org/10. 1016/j.jpeds.2016.06.078 